\title{
Abundances of Planetary Nebula NGC 5315 ^
}

\author{
S. R. Pottasch ${ }^{1}$, D. A. Beintema ${ }^{1,2}$, J. Bernard Salas ${ }^{1,2}$, J. Koornneef ${ }^{3}$, and W. A. Feibelman ${ }^{4}$ \\ 1 Kapteyn Astronomical Institute, PO Box 800, 9700 AV Groningen, The Netherlands \\ 2 SRON National Institute for Space Research, PO Box 800, 9700 AV Groningen, The Netherlands \\ 3 Infostrait bv, Postbus 901, 7301 BD Apeldoorn, The Netherlands \\ ${ }^{4}$ Laboratory for Astronomy and Solar Physics, Code 681, Goddard Space Flight Center, MD, USA
}

Received 13 May 2002 / Accepted 1 July 2002

\begin{abstract}
The ISO and IUE spectra of the elliptical nebula NGC 5315 is presented. These spectra are combined with the spectra in the visual wavelength region to obtain a complete, extinction corrected, spectrum. The chemical composition of the nebulae is then calculated and compared to previous determinations. The HST NICMOS observations of the nebula in 3 emission lines are also presented. These observations are used to determine the helium abundance as a function of position in the nebula. A discussion is given of possible evolutionary effects.
\end{abstract}

Key words. ISM: abundances - planetary nebulae: individual: NGC 5315 - infrared: ISM - ISM: lines and bands

\section{Introduction}

The southern planetary nebula NGC 5315 is a compact nebula with a diameter of about $4^{\prime \prime}$ at half power and $6^{\prime \prime}$ at the $1 \%$ level. The nebula is almost spherical (slightly elliptical) with a complicated structure, including a somewhat broken ring. The central star is clearly visible, having a visual magnitude of about 14. The star is classified as WC4 and hydrogen deficient (Mendez 1991). A P Cygni profile with a terminal velocity of about $3600 \mathrm{~km} \mathrm{~s}$ is measured in the $\mathrm{C}_{\text {Iv }}$ line (Feibelman 1998). This article reports and analyses two different kinds of observations of this nebula. First the ISO infrared spectrum is reported. It is combined with the IUE ultraviolet observations and the visible line spectrum to find the nebular abundances. The abundances found for the nebula are rather similar to those found in other PN: there is no indication that the nebula is hydrogen deficient. Since the nebula is formed by mass loss from the central star, and this star is hydrogen deficient, the problem arises: at what stage did this changeover take place. In particular, can this changeover be seen by looking with high spatial resolution at the appropriate lines in the nebula? To try to answer this question we have observed the nebula with the HST Nicmos instrument with a spatial resolution of better than $0.1^{\prime \prime}$ in order to study this problem.

Send offprint requests to: $\mathrm{S}$. R. Pottasch,

e-mail: pottasch@astro.rug.nl

* Based on observations with ISO, an ESA project with instruments funded by ESA Member States (especially the PI countries: France, Germany, The Netherlands and the UK) and with the participation of ISAS and NASA.

\subsection{Advantages of the ISO spectrum}

Including the ISO SWS spectra of planetary nebulae with spectra of the nebula in other spectral regions allows an abundance determination which has several important advantages. These have been discussed in earlier papers (e.g. see Pottasch \& Beintema 1999; Pottasch et al. 2000; or Bernard Salas et al. 2001).

The most important advantage is that the infrared lines originate from very low energy levels and thus give an abundance which is not sensitive to the temperature in the nebula, nor to possible temperature fluctuations. Furthermore, when a line originating from a high-lying energy level in the same ion is observed, it is possible to determine an effective temperature at which the lines of that particular ion are formed. When the effective temperature for many ions can be determined, it is possible to make a plot of effective temperature against ionization potential, which can be used to determine the effective temperature for ions for which only lines originating from a high energy level are observed. Use of an effective electron temperature takes into account the fact that ions are formed in different regions of the nebula. At the same time possible temperature fluctuations are taken into account.

Use of the ISO spectra have further advantages. One of them is that the number of observed ions used in the abundance analysis is approximately doubled, which removes the need for using large "Ionization Correction Factors", thus substantially lowering the uncertainty in the abundance. A further advantage is that the extinction in the infrared is almost negligible, eliminating the need to include large correction factors. 
Table 1. ISO observations of NGC 5315 (in units of $10^{-13} \mathrm{erg} \mathrm{cm}^{-2} \mathrm{~s}^{-1}$ ).

\begin{tabular}{|c|c|c|c|c|c|}
\hline Ident. & $\lambda(\mu \mathrm{m})$ & Intens. & Ident. & $\lambda(\mu \mathrm{m})$ & Intens. \\
\hline Н г 6-4 & 2.626 & 59 & {$\left[\mathrm{Fe}_{\mathrm{II}}\right]$} & 25.98 & 6.3 \\
\hline$?$ & 3.691 & 12 & [Si II $]$ & 34.81 & 64 \\
\hline Н І $5-4$ & 4.052 & 100 & {$[\mathrm{Fe}$ II $]$} & 35.35 & $<5$ \\
\hline$[\mathrm{Fe}$ II $]$ & 5.340 & $<2$ & [ $\mathrm{Ne}$ III] & 36.008 & 108 \\
\hline [Ar III] & 8.992 & 380 & {$\left[\mathrm{O}_{\mathrm{III}}\right]^{*}$} & 52 & 500 \\
\hline [S IV] & 10.551 & 310 & {$[\mathrm{~N} \text { III }]^{*}$} & 57 & 180 \\
\hline$?$ & 11.998 & 88 & {$\left[\mathrm{O}_{\mathrm{I}}\right]^{*}$} & 63 & 460 \\
\hline$[\mathrm{Ne}$ II $]$ & 12.813 & 510 & {$\left[\mathrm{O}_{\mathrm{III}}\right]^{*}$} & 88 & 78 \\
\hline [Ne III] & 15.55 & 1580 & {$\left[\mathrm{~N} \mathrm{II}^{*}\right.$} & 122 & 6.0 \\
\hline [S III] & 18.712 & 300 & {$\left[\mathrm{O}_{\mathrm{I}}\right]^{*}$} & 146 & 10 \\
\hline$[\mathrm{Fe}$ III] & 22.92 & 10.5 & {$\left[\mathrm{C}_{\mathrm{II}}\right]^{*}$} & 157 & 15.3 \\
\hline
\end{tabular}

* These data are from Liu et al. (2001).

\section{The spectrum of NGC 5315}

\subsection{ISO observations}

The ISO SWS observations were made with the SWS02 observing template which gives good spectral resolution for a limited number of lines. This was supplemented by an SWS01 observation. The intensity of the lines found in the spectrum is shown in Table 1 . The uncertainty of the stronger lines is about $10 \%$. The measurements were centered at $\mathrm{RA}(2000)$ $13^{\mathrm{h}} 53^{\mathrm{m}} 57.8^{\mathrm{s}}$ and $\operatorname{Dec}(2000)-66^{\circ} 30^{\prime} 50.2^{\prime \prime}$. As shall be shown from the HST observations, this position is about $4^{\prime \prime}$ to the east of the center of the nebula. But because the diaphragm used was $14^{\prime \prime} \times 20^{\prime \prime}$ below $12 \mu \mathrm{m}$ and somewhat larger above this wavelength, the entire nebula always was measured by the SWS. The LWS measurements of the entire nebula are reported and discussed by Liu et al (2001). That the SWS measurement includes the entire nebula can be checked by comparing the Balmer $\alpha$ line at $\lambda 4.052 \mu \mathrm{m}$ with that predicted by the total radio emission. Using a radio flux density of $415 \mathrm{mJy}$ (see below) a $\operatorname{Br} \alpha$ intensity of $1.04 \times 10^{-11} \mathrm{erg} \mathrm{cm}^{-2} \mathrm{~s}^{-1}$ is predicted, which agrees very well with the measured value.

\subsection{Comparison with other infrared observations}

A comparison is made in Table 2. Only five lines have been measured earlier and with different diaphragms. The IRAS measurements refer to the entire nebula, and this is probably the case for the measurements of Aitken \& Roche (1982) as their beam size is about the size of the nebula. There are two sets of IRAS data given in the table. The second set is a very recent reduction made in Groningen and is to be preferred. It is not clear why the earlier measurement of the S IV line was so high.

\subsection{Extinction}

The observed $6 \mathrm{~cm}$ radio emission $(415 \mathrm{mJy}$, Cahn et al. 1992) predicts a total $\mathrm{H} \beta$ flux for the nebula of $1.33 \times$ $10^{-10} \mathrm{erg} \mathrm{cm}^{-2} \mathrm{~s}^{-1}$. The observed $\mathrm{H} \beta$ is $3.8 \times 10^{-11}$ (Cahn et al. 1992). The extinction is therefore $c=0.54$. The observed
Table 2. NGC 5315: Comparison of infrared observations (in units of $10^{-13} \mathrm{erg} \mathrm{cm}^{-2} \mathrm{~s}^{-1}$ ).

\begin{tabular}{clrccc}
\hline \hline \multirow{2}{*}{$\begin{array}{c}\lambda \\
(\mu \mathrm{m})\end{array}$} & Ident. & ISO & \multicolumn{2}{c}{ IRAS } & AR \\
\cline { 4 - 5 } & & & $(1)$ & $(2)$ & $(3)$ \\
\hline 8.99 & {$\left[\mathrm{Ar}_{\mathrm{III}}\right]$} & 380 & $300:$ & 500 & 390 \\
10.51 & {$\left[\mathrm{~S}_{\mathrm{IV}}\right]$} & 310 & 850 & 335 & 290 \\
12.8 & {$\left[\mathrm{Ne}_{\mathrm{II}}\right]$} & 510 & 700 & 810 & 760 \\
15.55 & {$\left[\mathrm{Ne}_{\mathrm{III}}\right]$} & 1580 & 1700 & 2100 & \\
18.71 & {$\left[\mathrm{~S}_{\mathrm{III}}\right]$} & 300 & $300:$ & 270 & \\
\hline
\end{tabular}

(1) Pottasch et al. (1986); (2) Re-reduced measurements; (3) Aitken \& Roche (1982), 5.3" beam.

Table 3. NGC 5315: Hydrogen line intensities (in units of $10^{-12} \mathrm{erg} \mathrm{cm}^{-2} \mathrm{~s}^{-1}$ ).

\begin{tabular}{lcrrr}
\hline \hline$\lambda(\mu \mathrm{m})$ & Transit. & Obs. & Corr. & Theory \\
\hline 4.052 & $\operatorname{Br} \alpha(5-4)$ & 10. & 10.3 & 10.8 \\
2.626 & $\operatorname{Br} \beta(6-4)$ & 5.9 & 6.25 & 6.14 \\
$1.875^{*}$ & $\operatorname{Pa} \alpha(4-3)$ & 50. & 56. & 45.6 \\
$4861 \AA$ & $\mathrm{H} \beta$ & 38.1 & & 132. \\
\hline
\end{tabular}

The theoretical values assume an electron temperature for hydrogen of $T_{\mathrm{e}}=9000 \mathrm{~K}$.

${ }^{*}$ See Table 6.

Balmer decrement is consistent with this value: Cahn et al. (1992) give $c=0.60$. We will use $c=0.54$ or $E_{B-V}=0.37$. The extinction law used in this paper is taken from Fluks et al. (1994).

\subsection{Comparison of ISO and HST hydrogen lines with theory}

A comparison of the ISO and HST hydrogen line intensities with theory is made in Table 3 . This is useful to insure ourselves the ISO aperture covers the entire nebula and at the same time the extinction can be determined. As can be seen from the table, two infrared hydrogen lines can be seen in the ISO spectrum and one in the HST spectrum. There intensities are given in the third column of the table, and corrected for a very small extinction in the fourth column. The theoretical values are shown in the fifth column and are taken from Hummer \& Storey (1987) for $T_{\mathrm{e}}=9000 \mathrm{~K}$ and $N_{\mathrm{e}}=10^{4} \mathrm{~cm}^{-3}$. The values are scaled to give the best fit. At the same time this fixes the intensity of all other hydrogen lines, in particular the $\mathrm{H} \beta$ line. The intensity found for this line is in very good agreement with that found from the radio continuum emission. This indicates that the extinction is correct.

\subsection{The visual spectrum}

The visual spectrum has been measured by Torres-Peimbert \& Peimbert (1977), Pacheco et al. (1991) and Acker et al. (1989). Their measurements are shown in the first five columns of Table 4 for those lines which are of interest. In the last column 
Table 4. Visual spectrum of NGC 5315 (unreddened intensity in units of $10^{-12} \mathrm{erg} \mathrm{cm}^{-2} \mathrm{~s}^{-1}$ ).

\begin{tabular}{|c|c|c|c|c|c|}
\hline \multirow{2}{*}{$\begin{array}{c}\lambda \\
(\AA)\end{array}$} & \multirow[t]{2}{*}{ Ion } & \multicolumn{3}{|c|}{ Intensities } & \multirow{2}{*}{$\begin{array}{l}\text { Unred. } \\
\text { Intens. }\end{array}$} \\
\hline & & (1) & $(2)$ & (3) & \\
\hline 3727 & [O II] & 31 & & & 59.4 \\
\hline 3869 & [Ne III] & 43 & 28.2 & & 66.5 \\
\hline 4363 & [O III] & 3.3 & 3.5 & 3 & 5.2 \\
\hline 4711 & [Ar Iv] & 1.0 & & & 1.3 \\
\hline 4861 & $\mathrm{H} \beta$ & 100 & 100 & 100 & 132 \\
\hline 5007 & [O III] & 795 & 1101 & 831: & 1140 \\
\hline 5518 & [Cl II] & 0.33: & & 0.4 & 0.46 \\
\hline 5538 & [Cl II] & 1.1 & & 0.9 & 1.2 \\
\hline 5755 & [N II] & 7.6 & 4.6 & 5.0 & 5.7 \\
\hline 5876 & $\mathrm{He}_{\mathrm{I}}$ & 24 & 17.8 & 24 & 22.1 \\
\hline 6312 & [S III] & 5.8 & 3.4 & 4.0 & 4.0 \\
\hline 6563 & $\mathrm{H} \alpha$ & 483 & 490 & 340 & 380 \\
\hline 6584 & {$[\mathrm{~N}$ II] } & 306 & 291 & 266 & 250 \\
\hline 6717 & [S II] & 6.8 & & 6.0 & 5.2 \\
\hline 6731 & {$\left[\mathrm{~S}_{\mathrm{II}}\right]$} & 14.5 & & 6.0 & 10.3 \\
\hline 7136 & [Ar III] & 53.5 & 47.8 & 57. & 42.0 \\
\hline 7325 & [O II $]$ & 24.6 & 20.3 & 22. & 17.5 \\
\hline
\end{tabular}

(1) Torres-Peimbert \& Peimbert (1977), (2) Pacheco et al. (1991), (3) Acker et al. (1989).

the unreddened intensities are given, normalized to the total $\mathrm{H} \beta=1.33 \times 10^{-10} \mathrm{erg} \mathrm{cm}^{-2} \mathrm{~s}^{-1}$. An average value of the individual measurements is used, with the measurements of Torres-Peimbert \& Peimbert (1977) given double weight.

\subsection{The IUE ultraviolet spectrum}

The IUE spectrum has been discussed in detail by Feibelman (1998). He has observed the nebula with both high and low resolution spectra. This is important because the IUE diaphragm encompasses both the star and the nebula. Since the star has strong and broad emission lines, the stellar spectrum can be separated from the nebular spectrum only when high resolution spectra are available, clearly showing the broader stellar emission.

The stellar emission spectrum shows a much higher degree of ionization than the nebula. The star shows a strong line of ionized helium (He II) whereas the ion is not present (or in very small amounts) in the nebula. This is shown, e.g. by its absence in the visual spectrum. If He II is not present, we would not expect to see $\mathrm{O}$ IV either, since it has a very similar ionization potential. And indeed, the infrared line of [O IV] at $25.89 \mu \mathrm{m}$ is not present in the spectrum although it is one of the strongest lines in the infrared spectrum of nebulae showing He II. It is therefore unlikely that the lines at $\lambda 1400 \AA$ which Feibelman attributes to nebular $\mathrm{O}_{\mathrm{IV}}$ ] is due to this ion. In the same way it seems unlikely that the ions $\mathrm{Mg} \mathrm{v}$ and $\mathrm{Ne} \mathrm{v}$ are present in the nebula, since otherwise strong far infrared transitions are not seen.

The nebular IUE lines which will be used in the determination of abundances are listed in Table 5.

The first and second column of this table give the wavelength and identification of the observed lines. The third
Table 5. IUE spectrum of NGC 5315.

\begin{tabular}{llcccc}
\hline \hline \multirow{2}{*}{$\begin{array}{c}\lambda \\
(\AA)\end{array}$} & Ion & \multicolumn{3}{c}{ Intensities } & Unred. \\
\cline { 3 - 5 } & & $(1)$ & $(2)$ & $(3)$ & $(4)$ \\
\hline $1483^{\dagger *}$ & $\left.\mathrm{~N}_{\mathrm{IV}}\right]$ & 1.9 & & 2.1 & 3.4 \\
$1661^{*}$ & $\left.\mathrm{O}_{\mathrm{III}}\right]$ & 1.0 & & & \\
$1666^{\dagger}$ & $\left.\mathrm{O}_{\mathrm{III}}\right]$ & 2.85 & 4.66 & 4.2 & 6.3 \\
$1750^{\dagger *}$ & $\left.\mathrm{~N}_{\mathrm{III}}\right]$ & 4.73 & 6.65 & 6.0 & 8.5 \\
1892 & $\left.\mathrm{Si}_{\mathrm{III}}\right]$ & 2.9 & & 3.0 & 4.3 \\
1906 & $\left.\mathrm{C}_{\mathrm{III}}\right]$ & 8.2 & & & \\
$1909^{\dagger}$ & $\left.\mathrm{C}_{\mathrm{III}}\right]$ & 16.5 & 39.3 & 35. & 54.3 \\
$2325^{\dagger *}$ & $\left.\mathrm{C}_{\mathrm{II}}\right]$ & 6.2 & 8.9 & 7.8 & 14.8 \\
$2424^{\dagger} *$ & {$\left[\mathrm{Ne}_{\mathrm{IV}}\right]$} & 1.3 & 0.9 & 1.0 & 1.5 \\
2470 & {$\left[\mathrm{O}_{\mathrm{II}}\right]$} & 9.05 & 8.82 & 8.9 & 10.5 \\
\hline
\end{tabular}

(1) High dispersion - all intensity units $10^{-13} \mathrm{erg} \mathrm{cm}^{-2} \mathrm{~s}^{-1}$.

(2) Low resolution - all intensity units $10^{-13} \mathrm{erg} \mathrm{cm}^{-2} \mathrm{~s}^{-1}$.

(3) Average intensity used for the multiplet.

(4) Average intensities corrected for extinction, $E_{\mathrm{B}-\mathrm{V}}=0.37-$ all intensity units $10^{-12} \mathrm{erg} \mathrm{cm}^{-2} \mathrm{~s}^{-1}$.

${ }^{\dagger}$ The intensities given in Cols. 2-4 are for the entire multiplet.

${ }^{*}$ The intensities given in Col. 1 are for the entire multiplet.

column gives the intensity measured from the high resolution spectra. The fourth column is the intensity measured from the low resolution spectra and includes all nearby lines of the multiplet. The fifth column of the table gives the intensity used (for the entire multiplet). Finally, the last column gives the total intensity of the nebula corrected for extinction $E_{B-V}=0.37$.

\section{The HST observations of NGC 5315}

Observations of the nebula were made with NICMOS on 24 March 1998 in proposal 7837 of Pottasch and Koornneef. The purpose of the measurement was to see how the helium abundance varies as a function of position in the nebula. This can be done using only the neutral helium line because almost all helium is in the form of singly ionized helium. The absence of doubly ionized helium has been discussed in the previous section. There could be a small amount of neutral helium, since ions of similar ionization potential do exist. In Table 13 it can be seen that $\mathrm{C}^{+}$forms about $10 \%$ of the carbon, while $\mathrm{S}^{+}$is about $7 \%$ of the total sulfur abundance.

A helium variation might be expected since the central star has virtually no hydrogen in its atmosphere (e.g. Hamann 1996), while the nebula (which is formed from material ejected very recently by the star) is hydrogen rich (see Table 13). The spatial resolution of these measurements is sufficient for this purpose: the plate scale is $0.043^{\prime \prime} /$ pixel. The measurements were made by making use of narrow band filters which isolated individual lines: F187N isolated the hydrogen Paschen $\alpha(\mathrm{Pa} \alpha)$ line, F108N isolated the $\mathrm{He}_{\mathrm{I}}$ line at $\lambda 10830 \AA$ and F095N isolated the [S III] line at $\lambda 9531 \AA$. The purpose of measuring the $\left[\mathrm{S}_{\mathrm{III}}\right]$ line was to see whether this line has the same spatial behavior as the hydrogen line. In addition measurements were made in the continuum nearby the line so that the continuum (which was also observed through the line filter) could be subtracted. To this end, measurements were made using 
Table 6. Integrated line intensities: NGC 5315.

\begin{tabular}{llrr}
\hline \hline Line & $\lambda(\mu \mathrm{m})$ & Obs. & Corr. \\
\hline S $\left._{\text {III }}\right]$ & 0.9536 & 12.43 & 19.76 \\
$\mathrm{He}_{\mathrm{I}}$ & 1.0816 & 12.49 & 17.99 \\
$\mathrm{~Pa} \alpha$ & 1.8748 & 5.00 & 5.64 \\
\hline
\end{tabular}

Intensities in units of $10^{-11} \mathrm{erg} \mathrm{cm}^{-2} \mathrm{~s}^{-1}$.

Table 7. Integrated continuum intensities: NGC 5315.

\begin{tabular}{llrr}
\hline \hline$\lambda(\mu \mathrm{m})$ & $\delta \lambda(\mu \mathrm{m})$ & Obs. & Corr. \\
\hline 0.9715 & 0.0094 & 1.95 & 3.10 \\
1.1298 & 0.0110 & 1.66 & 2.39 \\
1.8986 & 0.0174 & 0.926 & 1.044 \\
\hline
\end{tabular}

Intensities in units of $10^{-14} \mathrm{erg} \mathrm{cm}^{-2} \mathrm{~s}^{-1} \AA^{-1}$.

Table 8. Continuum intensities from the central star of NGC 5315.

\begin{tabular}{ccrr}
\hline \hline$\lambda(\mu \mathrm{m})$ & $\delta \lambda(\mu \mathrm{m})$ & Obs. & Corr. \\
\hline 0.9536 & 0.0088 & 1.97 & 3.13 \\
1.1298 & 0.0110 & 2.03 & 2.93 \\
1.8986 & 0.0174 & 0.722 & 0.814 \\
\hline
\end{tabular}

Intensities in units of $10^{-15} \mathrm{erg} \mathrm{cm}^{-2} \mathrm{~s}^{-1} \AA^{-1}$.

the F097N, F113N and F190N filters which measured at $\lambda 9700$, 11300 and $19000 \AA$ A respectively. The continuum emission was only a few percent of the line emission, so the corrections were not large.

\subsection{Integrated intensities}

The filters used are quite narrow and contain only a single line. The total intensity of the three lines is given in Table 6 , integrated over a diameter of $7.7^{\prime \prime}$. The last column of the table gives the intensity after correction for extinction. This is the $\mathrm{Pa} \alpha$ line intensity used in Table 3 .

For completeness, the integrated nebular continuum intensities are given in Table 7 . These were measured with narrow band filters at the wavelengths given in the table and are integrated over a diameter of 7.7".

Using the same filters the radiation from the WC4 central star was measured. These values are shown in Table 8. The filter at $0.9715 \mu \mathrm{m}$ transmits the strong stellar C III line so that the value from a nearby filter which transmits mainly continuum is given.

\subsection{Comparison of images made in the three lines}

Four images of the nebula are shown as Fig. 1. Three of them of taken in the different lines $\mathrm{Pa} \alpha$, [S III], and $\mathrm{He}_{\mathrm{I}} \lambda 10830 \AA$. The fourth image shows the ratio of the $\mathrm{He}_{\mathrm{I}}$ line to $\mathrm{Pa} \alpha$. The cross at the center of the nebula is at the position $\mathrm{RA}(2000)=$ $13^{\mathrm{h}} 53^{\mathrm{m}} 57.036^{\mathrm{s}}$ and $\operatorname{Dec}(2000)=-66^{\circ} 30^{\prime} 50.15^{\prime \prime}$. The angle between the positive $y$ axis and north is $108^{\circ}$. It is clear from the figure that the $\left[\mathrm{S}_{\mathrm{III}}\right]$ image is very similar to that of $\mathrm{Pa} \alpha$. The helium line has a clearly different distribution.

\subsection{The radial distribution of the ions}

In order to study the global properties of the gas in the radial direction, the image is treated as spherically symmetrical; then we look for the effects of departures from this symmetry. While it is clear from Fig. 1 that this assumption is not completely correct, the geometry in the line-of-sight is not known and therefore will always remain an uncertainty. Therefore in Fig. 2 we have plotted the average intensity (in $\mathrm{mJy} / \mathrm{pixel}^{2}$ ) over a circular "shell" whose width is a single pixel, as a function of the distance from the central star. Each of the three profiles represents a single line $(\lambda 10830 \AA$, [S III] and $\mathrm{Pa} \alpha)$ since the continuum flux has been subtracted. This is the reason that the central star flux is not seen.

To see the quantitative result, the ratios of several lines, after converting mJy to intensity units, are given in Table 9, at four distances from the central star. In this table Cols. 2 through 7 give the line-of-sight values at the given radius, while the last column gives the local value at that radius. The ratio of the [S III] to $\mathrm{Pa} \alpha$ after correcting for the extinction is shown in Col. 2. In Col. 3 the ratio of $\mathrm{S}^{++} / \mathrm{H}$ is shown, using the method described in the following section. There is a slow variation of this ratio; it is unclear if this can be ascribed to a constant sulfur abundance coupled with a changing ionization state, or to a changing sulfur abundance. It is shown in the following section that $\mathrm{S}^{++}$is the predominant ionization state in the nebula, an order of magnitude more abundant than $\mathrm{S}^{+}$or $\mathrm{S}^{3+}$. For this reason there is a serious possibility that the sulfur abundance is increasing closer to the central star. The lower value at $0.75^{\prime \prime}$ may be caused by a line-of-sight effect. As will presently be discussed for the case of $\mathrm{He}$, there may be very little emitting material close to the central star, and what is observed at this position is the much stronger emission further out but in this line-of-sight.

The fourth column of Table 9 shows the $\lambda 10830 / \mathrm{Pa} \alpha$ ratio (corrected for extinction) while the fifth column gives the $\lambda 10830 / \mathrm{Pa} \beta$ ratio, which is found using the theoretical $\mathrm{Pa} \alpha / \mathrm{Pa} \beta$ ratio (Hummer \& Storey 1987). The determination of $\mathrm{He}^{+} / \mathrm{H}$ from $\lambda 10830 / \mathrm{Pa} \beta$ is difficult from theory. This is because there is still some question as to the accuracy of the theory. This can best be seen from Fig. 3, where the log of the ratio He I $\lambda 5876 / \mathrm{H} \beta$ has been plotted against $\lambda 10830 / \mathrm{Pa} \beta$. The open circles in the figure show all the planetary nebulae for which measurements have been made. The number of measurements of $\lambda 10830$ is a rather small because the wavelength is not accessible to many spectrographs. The primary references used are: Kelly \& Latter (1995). O’Dell (1963), Scrimger (1984) and Rudy et al. (1991a,b). The references for measurements of the $\lambda 5876 / \mathrm{H} \beta$ ratio are easily available in the literature. The extinction used is that given by the authors, but it is not critical because the ratios used are quite close in wavelength. The solid line shown on the figure is the least square best fit to the data and corresponds with $\lambda 10830 / \mathrm{Pa} \beta=45.6 \lambda 5876 / \mathrm{H} \beta$. 

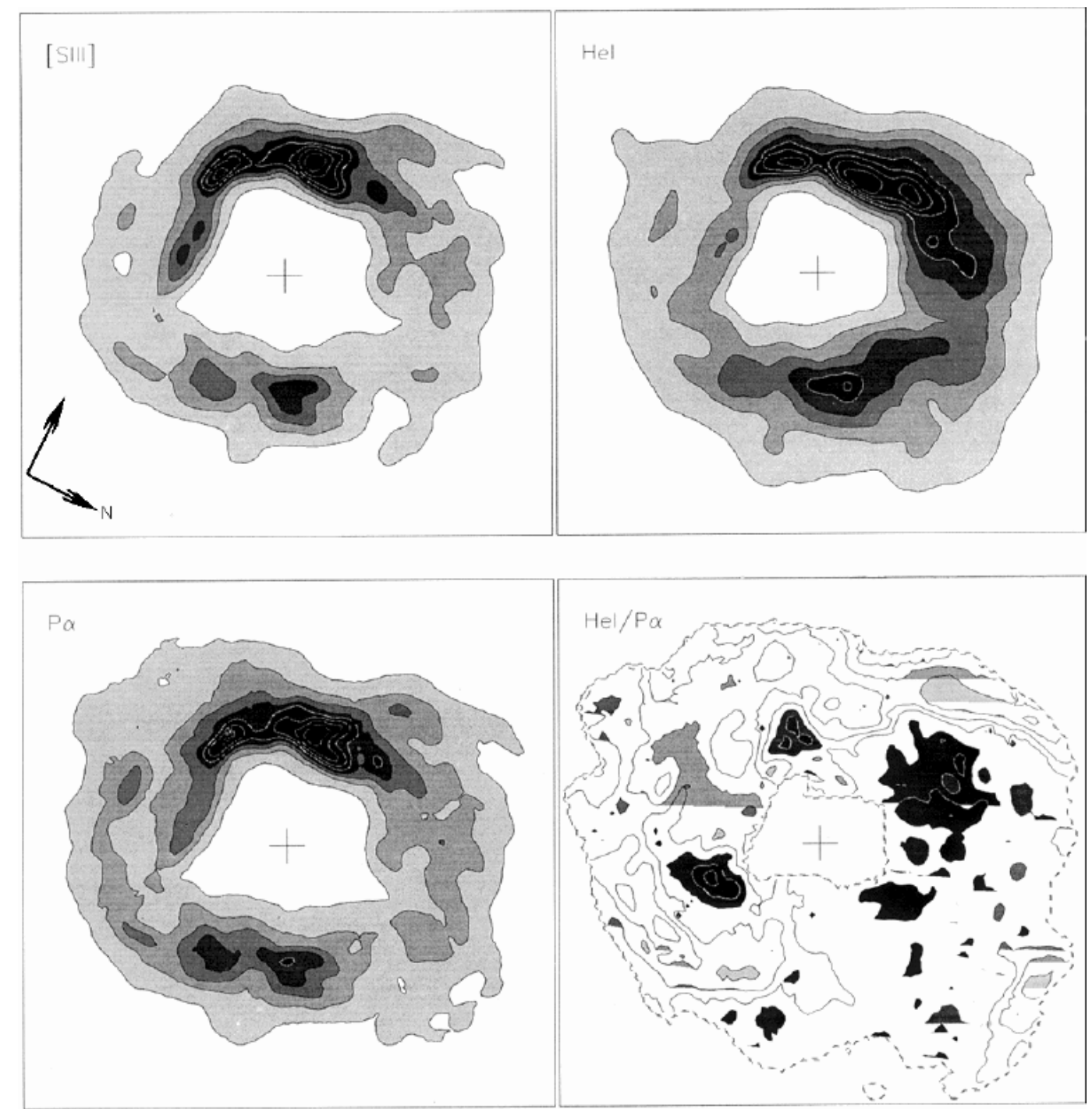

Fig. 1. Four images of NGC 5315 are shown. One is made in the light of the [S III] line, one in He $\mathrm{I}_{1} \lambda 10830 \AA$, one in Paschen $\alpha$ and the bottom right image is of the ratio of the helium line to the hydrogen $\mathrm{Pa} \alpha$ line. The width of the boxes is $4.7^{\prime \prime}$.

Table 9. Line intensities and nebular abundances as function of nebular radius.

\begin{tabular}{lcrrrrrr}
\hline \hline Radius & $\mathrm{S}$ III/P $\alpha$ & $\mathrm{S}^{++} / \mathrm{H}$ & $10830 / \mathrm{P} \alpha$ & $10830 / \mathrm{P} \beta$ & $5876 / \mathrm{H} \beta$ & $\mathrm{He} / \mathrm{H}$ & $\mathrm{He} / \mathrm{H}^{*}$ \\
\hline $0.75^{\prime \prime}$ & 3.01 & $0.738(-5)$ & 1.48 & 3.05 & 0.067 & 0.045 & \\
$1.0^{\prime \prime}$ & 4.52 & $1.11(-5)$ & 3.99 & 8.21 & 0.180 & 0.12 & 0.193 \\
$1.5^{\prime \prime}$ & 3.57 & $0.87(-5)$ & 3.28 & 6.75 & 0.148 & 0.099 & 0.109 \\
$2.0^{\prime \prime}$ & 3.18 & $0.78(-5)$ & 2.78 & 5.72 & 0.125 & 0.083 & 0.083 \\
\hline
\end{tabular}

${ }^{*}$ This helium to hydrogen ratio is corrected for line-of-sight integration (see text).

The other lines in Fig. 3 are from theory and are taken from Benjamin et al. (1999) which is the most complete theoretical discussion of the He I spectrum available. The dashed line is for $T_{\mathrm{e}}=10^{4} \mathrm{~K}$ and $N_{\mathrm{e}}=10^{4} \mathrm{~cm}^{-3}$, the dot dashed line corresponds to $T_{\mathrm{e}}=10^{4} \mathrm{~K}$ and $N_{\mathrm{e}}=10^{2} \mathrm{~cm}^{-3}$, the three dot dashed line is for $T_{\mathrm{e}}=2 \times 10^{4} \mathrm{~K}$ and $N_{\mathrm{e}}=10^{4} \mathrm{~cm}^{-3}$ and the dotted line corresponds to $T_{\mathrm{e}}=10^{4} \mathrm{~K}$ and $N_{\mathrm{e}}=10^{6} \mathrm{~cm}^{-3}$. It can be seen that it is possible to adjust $T_{\mathrm{e}}$ and $N_{\mathrm{e}}$ to obtain a line which is in agreement with the observations using reasonable values of $N_{\mathrm{e}}$ and $T_{\mathrm{e}}$. But, in spite of this agreement, there is something strange when comparing the results of Benjamin et al. (1999) with the observations. This is because using the theory and the observed individual values of temperature and density, one would expect larger variations as a function of $N_{\mathrm{e}}$ and $T_{\mathrm{e}}$ than are observed. The nebulae that have been plotted have values of $N_{\mathrm{e}}$ ranging from $3 \times 10^{3} \mathrm{~cm}^{-3}$ (NGC 3242 and NGC 6826) through $6 \times 10^{4} \mathrm{~cm}^{-3}$ (NGC 7027) and values of $T_{\mathrm{e}}$ between $9000 \mathrm{~K}$ and $18000 \mathrm{~K}$. It is expected from the calculations of Benjamin et al. (1999) that a much larger scatter of the points in Fig. 3 should result. However the observed points 


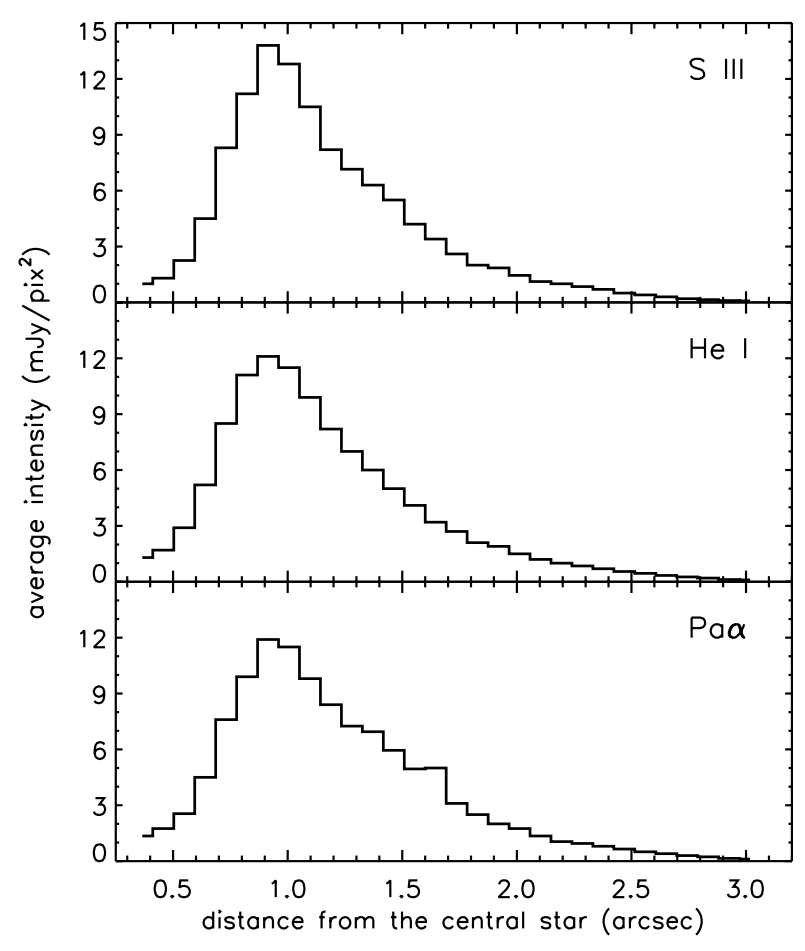

Fig. 2. The average intensity (assuming circular symmetry) is plotted as a function of the distance from the central star. The upper diagram is for [S III], the middle diagram for $\lambda 10830 \AA$ and the bottom diagram for $\operatorname{Pa} \alpha$.

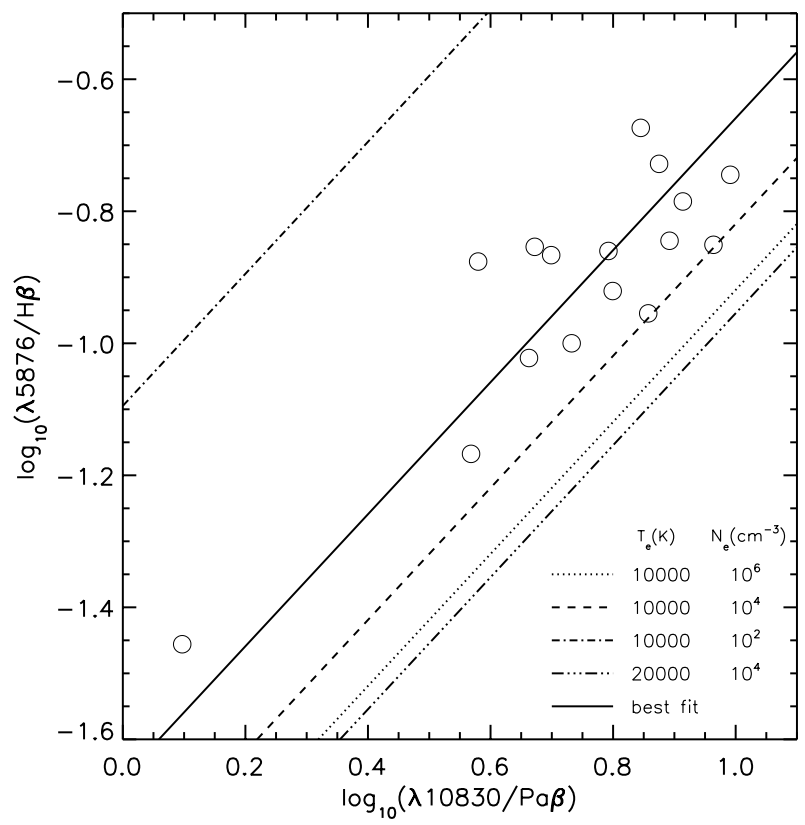

Fig. 3. The intensity ratio $\lambda 5876 / \mathrm{H} \beta$ is plotted as function of $\lambda 10830 / \mathrm{Pa} \beta$. Open circles give measured values with a best fit line through them. The other lines are theoretical values taken from Benjamin et al.

form a straight line with only a small scatter and apply to all observed nebulae. With this as background, we proceed in the following way. Using the observed value of $\lambda 10830 / \mathrm{Pa} \beta$, Fig. 3 is used to determine the value of $\lambda 5876 / \mathrm{H} \beta$ at that point using the heavy dashed (empirical) line. These values are shown in

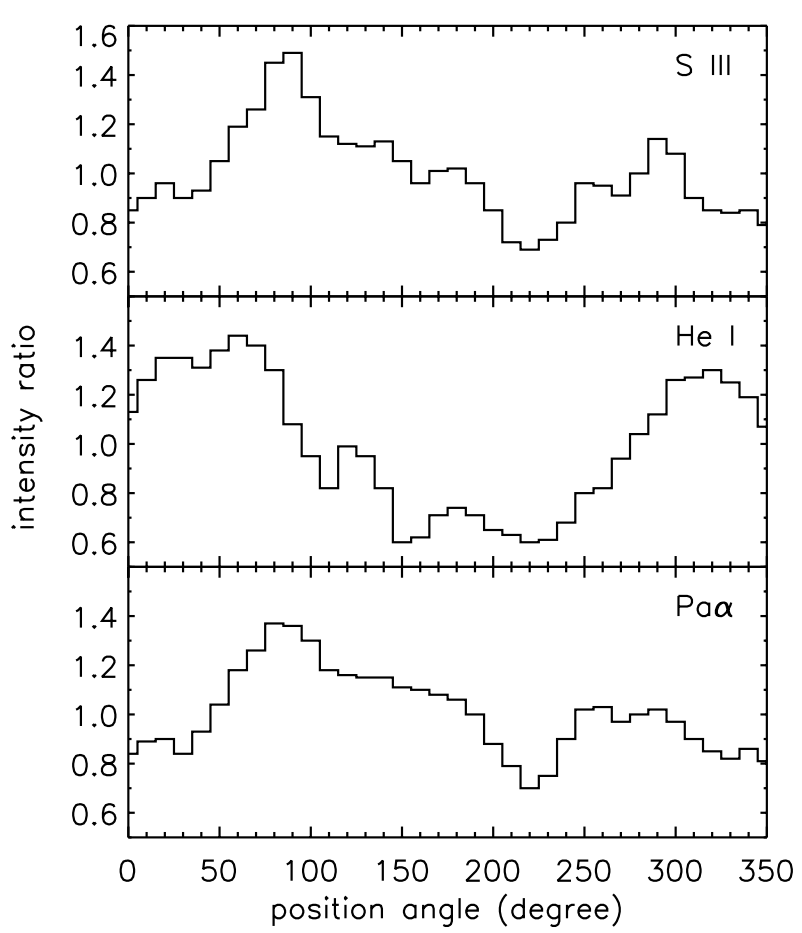

Fig. 4. The intensity integrated along the radial direction is plotted as a function of position angle at $10^{\circ}$ intervals. The intensity scale is relative to the average intensity. The upper diagram for the [S $\mathrm{III}]$ line, the middle diagram for $\mathrm{He}_{\mathrm{I}} \lambda 10830 \AA$, and the bottom diagram shows $\operatorname{Pa} \alpha$.

the 6th column of Table 9 and are in good agreement with the value 0.167 obtained from ground based spectra and previously given in Table 4 . The ground based measurement was made with a diaphragm of $2^{\prime \prime} \times 4^{\prime \prime}$ at a bright part of the nebula.

These values are then used to obtain the $\mathrm{He}^{+} / \mathrm{H}$ ratio using the theoretical predictions of Benjamin et al. (1999) for $T_{\mathrm{e}}=0.9 \times 10^{4} \mathrm{~K}$ and $N_{\mathrm{e}}=10^{4} \mathrm{~cm}^{-3}$. This value is also the $\mathrm{He} / \mathrm{H}$ ratio, since there is essentially no $\mathrm{He}^{++} \lambda 4686$ emission (and no O Iv $25.8 \mu \mathrm{m}$ as well). The helium abundance at the various distances are given in Col. 7 of Table 9. Because the nebula looks very much like a shell, we have made the assumption that the nebula can be divided into 10 concentric shells, each of which has a constant $\lambda 5876 / \mathrm{H} \beta$ ratio. The emission may then be integrated so that the three dimensional helium abundance can be obtained. This is shown in the last column of the table. There is so little emission closer to the star than $0.8^{\prime \prime}$ that no abundance is given there. It is clear that the helium abundance is increasing by an important amount as one goes from the outside of the nebula to $1.0^{\prime \prime}$ from the center. The exact values given in the table are influenced by the assumption made of spherical symmetry; the result of the change in helium abundance certainly remains in a real nebula.

\subsection{The azimuthal distribution of the ions}

It is clear from Fig. 1 that spherical symmetry is only useful as a first approximation. It can also be seen from this figure that the images in $\mathrm{Pa} \alpha$ and [S III] are quite similar, but differ from the He I image. To study this in more detail, the emission has 
Table 10. Helium to hydrogen ratios integrated over radius at a given position angle.

\begin{tabular}{lllcc}
\hline \hline PA $^{*}$ & $\frac{10830}{\mathrm{~Pa} \alpha}$ & $\frac{10830}{\mathrm{~Pa} \beta}$ & $\frac{5876}{\mathrm{H} \beta}$ & $\frac{\mathrm{He}}{\mathrm{H}}$ \\
\hline $28^{\circ}$ & 5.17 & 10.6 & 0.233 & 0.155 \\
$152^{\circ}$ & 1.71 & 3.52 & 0.077 & 0.051 \\
$319^{\circ}$ & 4.85 & 9.98 & 0.219 & 0.146 \\
\hline
\end{tabular}

${ }^{*}$ Position angle.

been integrated along the radial direction at various position angles. This is shown in Fig. 4, where the integrated intensity is plotted against the position angle at $5^{\circ}$ intervals for each of the three lines. In this figure the intensity is given with respect to the average intensity for the line (given in Table 6).

From the figure it can be seen that the integrated intensity varies as a function of position angle for all three of the lines. This variation is quite similar for $\mathrm{Pa} \alpha$ and the [S $\mathrm{III}]$ line, but quite different for the $\mathrm{He}_{\mathrm{I}}$ line. This is illustrated quantitatively in Table 10 where the ratio of the $\mathrm{He} \mathrm{I} \lambda 10830$ to $\mathrm{Pa} \alpha$ is listed in Col. 2 for three position angles representing extreme cases. It is seen that the differences can amount to a factor of 3 . This is a minimum effect because integration will always smooth the differences.

\subsection{Conclusion on the distribution of helium}

Various uncertainties surround the helium abundance determination. The first is the use of the $\lambda 10830$ line. The theoretical predictions for this line cannot be used, but the line intensity can be used to predict the intensity of the $\lambda 5876$ line, which then can be used to obtain the ionized helium abundance. The second uncertainty is whether the ionized helium represents the total helium abundance. This has been discussed above, where it was concluded that there is no doubly ionized helium, but there could be a small amount of neutral helium, of the order of 5 to $10 \%$. Within this uncertainty it appears that a shell of gas has been ejected with in general a higher helium abundance on the inside than on the outside. But the helium is not uniformly distributed and regions exist with a much higher helium abundance. Thus ejection of the helium rich material does not occur uniformly, but is a function of position on the star. Apparently either the ejection of material stopped at the moment very little hydrogen remained on the stellar surface, or at the moment the ejection stopped, some other process within the star occurred to make the surface helium rich.

\section{Chemical composition of the nebulae}

The method of analysis is the same as used in the papers cited in the introduction. First the electron density and temperature as function of the ionization potential is determined. Then the ionic abundances are determined, using density and temperature appropriate for the ion under consideration, together with Eq. (1). Then the element abundances are found for those elements in which a sufficient number of ions abundances have been determined.
Table 11. Electron density indicators.

\begin{tabular}{lcccc}
\hline \hline Ion & $\begin{array}{c}\text { Ioniz. } \\
\text { Pot.(eV) }\end{array}$ & $\begin{array}{c}\text { Lines } \\
\text { Used }\end{array}$ & $\begin{array}{c}\text { Observed } \\
\text { Ratio }\end{array}$ & $\begin{array}{c}N_{\mathrm{e}} \\
\left(\mathrm{cm}^{-3}\right)\end{array}$ \\
\hline $\mathrm{S}_{\text {II }}$ & 10.4 & $6731 / 6716$ & 2.0 & 10000 \\
$\mathrm{O}_{\text {II }}$ & 13.6 & $7325 / 3727$ & 0.30 & 18000 \\
$\mathrm{~S}_{\text {III }}$ & 23.3 & $9536 / 18.7$ & 5.2 & 26000 \\
$\mathrm{Cl}_{\text {III }}$ & 23.8 & $5538 / 5518$ & & $23500^{\dagger}$ \\
$\mathrm{C}_{\text {III }}$ & 24.4 & $1907 / 1909$ & 0.5 & 60000 \\
$\mathrm{O}_{\text {III }}$ & 35.1 & $52 / 88$ & 4.3 & 2300 \\
$\mathrm{Ar}_{\text {IV }}$ & 40.7 & $4740 / 4711$ & & $12000^{\dagger}$ \\
$\mathrm{Ne}_{\text {III }}$ & 41.0 & $15.5 / 36.0$ & 14.6 & 14000 \\
\hline
\end{tabular}

$\dagger$ From Liu et al. (2001).

\subsection{Electron density}

The ions used to determine $N_{\mathrm{e}}$ are listed in the first column of Table 11. The ionization potential required to reach that ionization stage, and the wavelengths of the lines used, are given in Cols. 2 and 3. Note that the wavelength units are $\AA$ when 4 ciphers are given and microns when 3 ciphers are shown. The observed ratio of the lines is given in the fourth column; the corresponding $N_{\mathrm{e}}$ is given in the fifth column. The temperature used is discussed in the following section, but is unimportant since these line ratios are essentially determined by the density. There are not so many density determinations because sensitive measurements in the visible are rare. For $\mathrm{Cl}_{\mathrm{III}}$ and $\mathrm{Ar}$ IV the results reported by Liu et al. (2001) are given.

There is no indication that the electron density varies with ionization potential in a systematic way. As already pointed out by Liu et al. (2001) the [O III] lines always give a lower density than the other lines. Ignoring these lines, the electron density appears to be between 15000 and $20000 \mathrm{~cm}^{-3}$. It is interesting to compare this value of the density with the rms density found from the $\mathrm{H} \beta$ line. This depends on the distance of the nebula which isn't accurately known, and on the angular size of the nebula. For this calculation we shall use a distance of $2 \mathrm{kpc}$ (Acker et al. 1992). A sphere of diameter of $4^{\prime \prime}$ will represent the nebula. The $\mathrm{H} \beta$ flux has been given above and the electron temperature will be discussed below. We obtain a value of $24000 \mathrm{~cm}^{-3}$. This value is uncertain because the distance is not well known. However the rms density varies only as the square root of the distance. It is therefore likely that the similarity of these values to the forbidden line densities is real. This probably indicates inhomogeneities do not play a dominant role in determining the density. We will use the forbidden line densities in further discussion of the abundances.

\subsection{Electron temperature}

A number of ions have lines originating from energy levels far enough apart that their ratio is sensitive to the electron temperature. These are listed in Table 12, which is arranged similarly to the previous table. The electron temperature remains roughly constant as a function of ionization potential. This is in contrast to what has been found in most other nebulae with ISO observations discussed previously, although in NGC 6537 
Table 12. Electron temperature indicators.

\begin{tabular}{lcccc}
\hline \hline Ion & $\begin{array}{c}\text { Ioniz. } \\
\text { Pot. }(\mathrm{eV})\end{array}$ & $\begin{array}{c}\text { Lines } \\
\text { Used }\end{array}$ & $\begin{array}{c}\text { Observed } \\
\text { Ratio }\end{array}$ & $\begin{array}{c}T_{\mathrm{e}} \\
(\mathrm{K})\end{array}$ \\
\hline $\mathrm{N}_{\text {II }}$ & 14.5 & $5755 / 6584$ & 0.0255 & 9300 \\
$\mathrm{~S}_{\text {III }}$ & 23.3 & $6312 / 18.7$ & 0.13 & 9600 \\
$\mathrm{Ar}_{\text {III }}$ & 27.6 & $7136 / 8.99$ & 1.1 & 9300 \\
$\mathrm{~N}_{\text {III }}$ & 29.6 & $1750 / 57$ & 0.47 & 8300 \\
$\mathrm{O}_{\text {III }}$ & 35.1 & $4363 / 5007$ & 0.0046 & 8500 \\
$\mathrm{O}_{\text {III }}$ & 35.1 & $1663 / 5007$ & 0.0055 & 9500 \\
$\mathrm{O}_{\text {III }}$ & 35.1 & $5007 / 52$ & 22.8 & 9200 \\
$\mathrm{Ne}_{\text {III }}$ & 41.0 & $3868 / 15.5$ & 0.42 & 8700 \\
\hline
\end{tabular}

the electron temperature was also found to remain constant. In the previous nebulae (including NGC 6537) the temperature found from the [Ne III] lines is usually lower than what would be expected from the ions with a similar ionization potential. This does not appear to be the case for NGC 5315. Thus a value of electron temperature of $9100 \mathrm{~K}$ is used for all ionization stages observed.

\subsection{Ionic and element abundances}

The ionic abundances have been determined using the following equation:

$\frac{N_{\text {ion }}}{N_{\mathrm{p}}}=\frac{I_{\text {ion }}}{I_{\mathrm{H}_{\beta}}} N_{\mathrm{e}} \frac{\lambda_{\mathrm{ul}}}{\lambda_{\mathrm{H}_{\beta}}} \frac{\alpha_{\mathrm{H}_{\beta}}}{A_{\mathrm{ul}}}\left(\frac{N_{\mathrm{u}}}{N_{\text {ion }}}\right)^{-1}$

where $I_{\mathrm{ion}} / I_{\mathrm{H}_{\beta}}$ is the measured intensity of the ionic line compared to $\mathrm{H} \beta, N_{\mathrm{p}}$ is the density of ionized hydrogen, $\lambda_{\mathrm{ul}}$ is the wavelength of this line, $\lambda_{\mathrm{H}_{\beta}}$ is the wavelength of $\mathrm{H} \beta, \alpha_{\mathrm{H}_{\beta}}$ is the effective recombination coefficient for $\mathrm{H} \beta, A_{\mathrm{ul}}$ is the Einstein spontaneous transition rate for the line, and $N_{\mathrm{u}} / N_{\text {ion }}$ is the ratio of the population of the level from which the line originates to the total population of the ion. This ratio has been determined using a five level atom.

\subsubsection{NGC 5315}

The results are given in Table 13, where the first column lists the ion concerned, and the second column the line used for the abundance determination. The third column gives the intensity of the line used relative to $\mathrm{H} \beta$. The fourth column gives the ionic abundances, and the fifth column gives the Ionization Correction Factor (ICF). This has been determined empirically. Notice that the ICF is usually small, less than a factor 1.5, and the element abundances, given in the last column, are probably well determined.

\section{Comparison with other abundance determinations}

Table 14 shows a comparison of our abundances with the most important determinations in the past 20 years. There is reasonable agreement, usually to within a factor of two. This is the first time the carbon abundance has been determined, and the
Table 13. Ionic concentrations and chemical abundances in NGC 5315. Wavelength in Angstrom for all values of $\lambda$ above 1000, otherwise in $\mu \mathrm{m}$.

\begin{tabular}{lccccc}
\hline \hline Ion & $\lambda$ & Intens. & $N_{\text {ion }} / N_{\mathrm{p}}$ & ICF & $N_{\text {el. } / N_{\mathrm{p}}}$ \\
\hline $\mathrm{He}^{+}$ & 5875 & 0.186 & 0.124 & 1 & 0.124 \\
$\mathrm{C}^{+}$ & 2325 & 0.122 & $4.65(-5)$ & & \\
$\mathrm{C}^{++}$ & 1909 & 0.418 & $2.6(-4)$ & 1.4 & $4.4(-4)$ \\
$\mathrm{N}^{+}$ & 6584 & 1.9 & $5.4(-5)$ & & \\
$\mathrm{N}^{++}$ & 1750 & 0.065 & $2.0(-4)$ & & \\
$\mathrm{N}^{+3}$ & 1487 & 0.026 & $1.3(-4)$ & 1 & $4.6(-4)$ \\
$\mathrm{O}^{+}$ & 3727 & 0.45 & $7.1(-5)$ & & \\
$\mathrm{O}^{++}$ & 5007 & 8.46 & $4.5(-4)$ & 1 & $5.2(-4)$ \\
$\mathrm{Ne}^{+}$ & 12.8 & 0.386 & $6.4(-5)$ & & \\
$\mathrm{Ne}^{++}$ & 15.5 & 1.2 & $9.0(-5)$ & & \\
$\mathrm{Ne}^{+3}$ & 2425 & 0.012 & $5.0(-6)$ & 1 & $1.6(-4)$ \\
$\mathrm{S}^{+}$ & 6731 & 0.078 & $8.5(-7)$ & & \\
$\mathrm{S}^{++}$ & 18.7 & 0.227 & $5.7(-6)$ & & \\
$\mathrm{S}^{++}$ & 6312 & 0.030 & $1.0(-5)$ & & \\
$\mathrm{S}^{++}$ & 9531 & 1.47 & $1.1(-5)$ & & \\
$\mathrm{S}^{+3}$ & 10.5 & 0.24 & $1.1(-6)$ & 1 & $1.2(-5)$ \\
$\mathrm{Ar}^{++}$ & 8.99 & 0.29 & $3.3(-6)$ & & \\
$\mathrm{Ar}^{++}$ & 7136 & 0.32 & $3.8(-6)$ & & \\
$\mathrm{Ar}^{+3}$ & 4741 & 0.010 & $7.2(-7)$ & 1.1 & $4.6(-6)$ \\
$\mathrm{Fe}^{+}$ & 25.98 & 0.0048 & $1.1(-7)$ & & \\
$\mathrm{Fe}^{++}$ & 22.92 & 0.008 & $2.7(-7)$ & & \\
$\mathrm{Fe}^{+4}$ & 20.8 & $<0.003$ & $<1.5(-7)$ & 1.4 & $5.3(-7)$ \\
$\mathrm{Si}^{+}$ & 34.8 & 0.049 & $8.6(-6)$ & & \\
$\mathrm{Si}^{++}$ & 1892 & 0.033 & $3.3(-6)$ & & \\
\hline
\end{tabular}

Intensities given with respect to $\mathrm{H} \beta=1$.

nebula appears to have similar amounts of carbon and oxygen. The nitrogen is also approximately the same as oxygen, which agrees with some, but not all, of the earlier determinations. The iron abundance has been determined for the first time in this nebula and is quite reliable. It is about a factor of 60 lower than the solar abundance (Anders \& Grevesse 1989; Grevesse \& Noels 1993), and this may be explained by the iron being mostly in the form of dust. This was also found in NGC 6302.

The helium abundance has been discussed earlier. Only the $\lambda 5875 \AA$ line was used, because, as discussed above, the theoretical determination of this line is the most reliable. It is possible that a small correction must be made for neutral helium. This could increase the helium abundance by at most $10 \%$.

The present results indicate that oxygen is slightly more abundant than carbon. This is consistent with the absence of PAH emission in the infrared spectrum.

\subsection{Errors}

It would be interesting to determine the errors in the abundance determination. Unfortunately this is difficult to do. The reason for this is the following. The error can occur at several stages in the determination. An error can occur in the intensity determination and this can be specified: it is probably less than $30 \%$ and may be lower for the stronger lines. An error may occur in correcting for the extinction, either because the extinction is incorrect or the average reddening law is not applicable. 
Table 14. Comparison of abundances in NGC 5315.

\begin{tabular}{|c|c|c|c|c|c|c|}
\hline Elem. & Present & ТPP(1) & $\mathrm{KB}(2)$ & $\mathrm{FP}(3)$ & SKAS(4) & Malkov(5) \\
\hline $\mathrm{He}$ & 0.124 & 0.122 & 0.091 & 0.106 & 0.11 & 0.11 \\
\hline $\mathrm{C}$ & $4.4(-4)$ & & & & & \\
\hline $\mathrm{N}$ & $4.6(-4)$ & $6.7(-4)$ & $6.1(-4)$ & $2.7(-4)$ & $2.3(-4)$ & $22.5(-4)$ \\
\hline $\mathrm{O}$ & $5.2(-4)$ & $7.1(-4)$ & $6.4(-4)$ & $7.6(-4)$ & $3.9(-4)$ & $4.7(-4)$ \\
\hline$S$ & $1.2(-5)$ & & & $1.9(-5)$ & $1.4(-5)$ & $3.1(-6)$ \\
\hline $\mathrm{Ar}$ & $4.6(-6)$ & & & $4.8(-6)$ & $6.5(-6)$ & $3.0(-6)$ \\
\hline $\mathrm{Ne}$ & $1.6(-4)$ & $1.9(-4)$ & $1.9(-4)$ & $1.1(-4)$ & & $1.2(-4)$ \\
\hline $\mathrm{Fe}$ & $5.3(-7)$ & & & & & \\
\hline
\end{tabular}

(1) Torres-Peimbert \& Peimbert (1977); (2) Kingsburgh \& Barlow (1994); (3) de Freitas Pacheco et al. (1991); (4) Samland et al. (1992); Malkov (1998).

We have tried to minimize this possibility by making use of known atomic constants to relate the various parts of the spectrum. Thus the ratio of $\operatorname{Br} \alpha$ to $\mathrm{H} \beta$ is an atomic constant. The ratio of the infrared spectrum to the visible spectrum is fixed in this way.

A further error is introduced by the correction for unseen stages of ionization. This varies with the element, but is usually small because very many ionization stages are observed. Thus for neon all but neutral neon is observed, so that the error will be small. This is also true for sulfur, argon, oxygen and nitrogen where the higher stages of ionization which are not observed contribute very little to the abundance.

There is also an error due to uncertainties in the collisional cross-section and the transition probabilities. The former is estimated at $25 \%$ and the latter is smaller. One might think that when comparing nebulae this error will disappear, since the same error is made in all nebulae. This is partly true, but because the contribution of a given ion varies with the nebula, some unspecifiable error will remain. In a previous article this has been estimated as 30\% (Pottasch et al. 2001), but this may be an overestimate.

\section{The central star}

As mentioned in Sect.1, this nebula is excited by a WC4 central star. Using the visual magnitude of 14.2 listed by Kaler \& Jacoby (1991) which they obtain by averaging various rather different values found in the literature and the $\mathrm{H} \beta$ flux given above, the hydrogen Zanstra temperature $\left(T_{\mathrm{z}}(\mathrm{H})\right)$ is about $66000 \mathrm{~K}$. No doubly ionized helium is seen in the nebula; this is confirmed by the absence of any noticeable O Iv emission. This is consistent with the above temperature. The "Stoy" or Energy Balance temperature can also be found from the above data. The value of the ratio of "forbidden line emission" (including all collisionally excited emission) to $\mathrm{H} \beta$ is 16.7 , which leads to an energy balance temperature $\left(T_{\mathrm{EB}}\right)$ of $54000 \mathrm{~K}$. These values are in reasonable agreement with those given by Kaler \& Jacoby (1991) of $T_{\mathrm{EB}}=56000 \mathrm{~K}$ and $T_{\mathrm{z}}(\mathrm{H})=65000 \mathrm{~K}$. Kaler \& Jacoby however also give a He II Zanstra temperature of $T_{\mathrm{Z}}(\mathrm{He}$ II $)=82000 \mathrm{~K}$ for which they give more weight. We believe that this is wrong and that they have mistaken the stellar $\lambda 4686$ emission for nebular emission.

\section{Conclusions}

If all of the hydrogen-rich envelope of the AGB star is ejected and, as in this case, a hydrogen-deficient star remains, it might be expected that some of the hydrogen-deficient material would be ejected as well. We have investigated the abundances in NGC 5315 with this in mind. Such a scenario would lead to an increased abundance of helium, carbon and oxygen, and a decrease in nitrogen abundance. This is not seen. The carbon abundance is similar to other PN investigated (Pottasch et al. 2001) as is the oxygen abundance as well. The nitrogen abundance is certainly not lower than other PN, rather it is on the high side. This indicates that the nitrogen (and the somewhat high helium abundance) was created in a dredge-up event which brought it to the surface, just as in PN which do not have WR central stars. The star apparently knows how to stop mass loss before the hydrogen-poor material is expelled.

In order to investigate whether any abundance changes can be seen in the nebula we report on HST NICMOS images made in hydrogen, helium and sulfur lines. These were made with a resolution of $0.043^{\prime \prime}$ to see whether any abundance changes occur within the nebula. The image in [S III] looks very similar to that of $\operatorname{Pa} \alpha$, consistent with the conclusion that the nebula consists of hydrogen rich material with a constant sulfur abundance. On the other hand the He I image is substantially different from the hydrogen image, indicating a buildup of helium in certain positions. It is likely that helium rich material is being ejected in two nearly opposite directions. How this occurs, and why it is not seen in the sulfur, is not known.

The buildup of infrared continuum emission is predominately longward of about $14 \mu \mathrm{m}$. Some dust features are seen, most important are the silicate emission at about $11.3 \mu \mathrm{m}$ and a broader feature at $33.5 \mu \mathrm{m}$. As mentioned above, PAH features are absent or very small. This is consistent with the oxygen rich nature of the nebula.

\section{References}

Acker, A., Ochsenbein, F., Stenholm, B., et al. 1992, Strasb.-ESO Catalogue

Acker, A., Köppen, J., Stenholm, B., \& Jasniewicz, G. 1989, A\&AS, 80, 201

Aitken, D. K., \& Roche, P. F. 1982, MNRAS, 200, 217

Anders, E., \& Grevesse, N. 1989, Geochem. Cosmo., 53, 197 
Benjamin, R. A., Skillman, E. D., \& Smits, D. P. 1999, ApJ, 514, 307

Bernard Salas, J., Pottasch, S. R., Beintema, D. A., \& Wesselius, P. R. 2001, A\&A, 367, 949

Bernard Salas, J., Pottasch, S. R., Feibelman, W. A., \& Wesselius, P. R., 2002, A\&A, 387, 301

Cahn, J. H., Kaler, J. B., \& Stanghellini, L. 1992, A\&AS, 94, 399

Feibelman, W. A. 1998, ApJ, 506, 773

Fluks, M. A., Plez, B., de Winter, D., et al. 1994, A\&AS, 105, 311

Grevesse, N., \& Noels, A. 1993, in Origin of the Elements, ed. N. Prantos, E. Vangioni-Flam, \& M. Casse (Cambridge University Press), 15

Hamann, W.-R. 1996, Ap\&SS, 238, 31

Hummer, D. G., \& Storey, P. J. 1987, MNRAS, 224, 801

Kaler, J. B., \& Jacoby, G. H. 1991, ApJ, 372, 215

Kelly, D. M., \& Latter, W. B. 1995, AJ, 109, 1320

Kingsburgh, R. L., \& Barlow, M. J. 1994, MNRAS, 271, 257

Liu, X.-W., Barlow, M. J., Cohen, M., et al. 2001, MNRAS, 323, 343

Malkov, Yu, F. 1998, Astron. Rep., 42, 293

Mendez, R. H. 1992, IAU Symp., 145, 375
O’Dell, C. R. 1963, ApJ, 138, 1018

Pacheco, J. A. de Freitas, Maciel, W. J., Costa, R. D. D., \& Barbury, B. 1991, A\&A, 250, 159

Pottasch, S. R., \& Beintema, D. A. 1999, A\&A, 347, 974

Pottasch, S. R., Beintema, D. A., \& Feibelman, W. A. 2000, A\&A, 363, 767

Pottasch, S. R., Beintema, D. A., Bernard Salas, J., \& Feibelman, W. A. 2001, A\&A, 380, 684

Pottasch, S. R., Preite-Martinez, A., Olnon, F. M., Mo, J.-E., \& Kingma, S. 1986, A\&A, 161, 363

Rowlands, N., Houck, J. R., Skrutskie, M. F., \& Shure, M. 1993, PASP, 105,1287

Rudy, R. J., Rossano, G. S., Erwin, P., et al. 1991a, ApJ, 368, 468

Rudy, R. J., Cohen, R. D., Rossano, G. S., et al. 1991b, ApJ, 380, 151

Samland, M., Koppen, J., Acker, A., \& Stenholm, B. 1992, A\&A, 264, 184

Scrimger, J. N. 1984, ApJ, 280, 170

Torres-Peimbert, S., \& Peimbert, M. 1977, Rev. Mex. Astron. Astrofis., 2, 181 